\title{
Satellite Links Integrated in 5G SDN-enabled Backhaul Networks: An Iterative Joint Power and Flow Assignment
}

\author{
Eva Lagunas*, Lei Lei, Symeon Chatzinotas, Björn Ottersten \\ Interdisciplinary Centre for Security, Reliability and Trust (SnT), University of Luxembourg \\ Email: \{eva.lagunas,lei.lei,symeon.chatzinotas,bjorn.ottersten\}@uni.lu \\ ${ }^{*}$ Corresponding author
}

\begin{abstract}
While recent technological advances have focused on how to provide increased data rate in 5G Radio Access Network (RAN), very few attention has been paid on how the current microwave backhaul network can handle the transport of such huge traffic flows. In this paper, we address two key aspects for the efficient adaptation of the backhaul network to the upcoming SDN-enabled 5G wireless communications. First, we consider the availability of dedicated satellite links to off-load the traffic from the terrestrial backhaul links. These satellite links operate on the non-exclusive Ka band, which is shared with the terrestrial microwave backhaul links. Second, and given the interference limited scenario, we address the power control and flow assignment of the resulting satelliteterrestrial network. While most of the flow assignment works consider the link rates as fixed and given, here we provide a novel formulation which links the achievable link rates with the assigned transmission power. In particular, we propose an iterative joint power control and flow assignment which takes into account the long propagation delay imposed by the satellite links. We transform the resulting non-convex problem into a Geometric Programming (GP) problem, which can be optimally solved in an efficient way. Simulation results validate and demonstrate the benefits of the proposed approach.
\end{abstract}

\section{INTRODUCTION}

The existing backhaul network might run out of capacity in the future due to the significantly increasing data traffic expected with $5 \mathrm{G}$, with catastrophic consequences in terms of performance of regular mobile services. While fiber is the most promising solution in terms of capacity, its deployment is limited to higher populated areas. To serve sites that may not have easy or cost-effective access to fiber, microwave continues to be the best choice. In fact, according to [1], 40\% of backhaul connections are expected to be based on wireless technology by 2023. Conventionally allocated in the $19 \mathrm{GHz}$ band, and rarely going over $30 \mathrm{GHz}$, microwave links usually cover short-range links up to few tens of kilometers, thus requiring a mesh topology to interconnect the Radio Access Network (RAN) with the backbone of the network.

The distribution of the different traffic flows over multihop networks has been extensively studied in the literature [2]-[4]. Among the most popular approaches are the ones based on the shortest path (path with least number of hops) or the ones based on high throughput end-to-end capacity. In spectrum sharing scenarios, interference becomes a major concern in the link performance [5], [6]. In this context, smart
Radio Resource Management (RRM) comes into play, since the interference level depends on how the radio resources are allocated. Therefore, in this paper, we address the joint optimization of flow assignment and the management of radio resources such as the transmit power.

The proposed framework considers two main peculiarities. First is the introduction of satellite links to support the conventional terrestrial wireless backhauling network. Satellite backhauling links has been shown to provide additional capability and flexibility to the conventional backhauling infrastructure [7], [8]. In addition, satellite becomes the de facto solution to extend the coverage to more remote regions and may generate cost savings in infrastructure deployment for lowtraffic zones or simply where deployment of fixed towers is cost-prohibitive. The centralized management of the satelliteterrestrial network is possible thanks to the emerging Software Defined Network (SDN) technology [9]. Second is the shared spectrum usage between satellite and terrestrial links. The satellite industry is seeking to maximize its spectrum usage and investing to improve spectrum efficiency. In this context, the non-exclusive Ka-band (17.7-19.7 GHz for Space-to-Earth and 27.5-29.5 GHz for Earth-to-Space) has been identified as the most promising band [10], [11].

The problem of flow assignment combined with RRM has received some attention in the literature. Most of them have focus on general terrestrial backhaul networks, e.g. [12]-[14]. [14] proposes a new approach which makes flow assignment and resource allocation decisions based on node location information solely, while Code-Division Multiple Access (CDMA) wireless networks are considered in [13] and [12]. The inclusion of satellite links has been previously addressed in [15] and [11], the former without the spectrum sharing assumption, i.e. without interference, and the latter with spectrum sharing but targeting the design of the frequency allocation and scheduling of the transmissions in the time domain.

Unlike previous works, this paper considers the spectral reuse of the non-exclusive Ka-band and provides the optimal power control strategy and flow assignment that maximizes the sum throughput of the overall backhaul network, while restricting the use of the satellite link whenever possible in order to avoid the expensive cost of satellite bandwidth. Due to the non-linearity of the throughput expression and the presence 
of interference, we propose to use a high Signal-to-Interference Ratio (SIR) approximation which leads to a Geometric Programming (GP) problem. With a simple logarithmic change of variable, the GP problem is transformed into a convex optimization, which can be efficiently solved providing the global optimal solution.

\section{System Model}

Consider a backhaul topology modeled as a directed graph ( $N, L)$, consisting of $N$ nodes and $L$ links. Most of the nodes are terrestrial only, while few of them are equipped with satellite connectivity as well. The latter are referred as hybrid nodes.

The network topology is fully represented by the node-link incidence matrix $\mathbf{A} \in \mathbb{R}^{N \times L}$ defined as,

$$
A(\ell, n)= \begin{cases}-1 & \text { if } \ell \in \mathcal{O}(n), \\ 1 & \text { if } \ell \in \mathcal{I}(n), \\ 0 & \text { if otherwise }\end{cases}
$$

where $\mathcal{O}(n)$ denotes the set of links outgoing from node $n$, and $\mathcal{I}(n)$ denotes the set of links incoming to node $n$.

We assume that each node can inject data into the network and received data from multiple sources. In order to model the data flow in the network, we define the source vector $\mathbf{s}^{(d)} \in \mathbb{R}^{N}$ whose entry $s^{(d)}(n)$ denotes the non-negative flow injected into the network at node $n, n \neq d$ and intended for node $d$. The component $s^{(d)}(d)$ is defined as $-\sum_{n \neq d} s^{(d)}(n)$ so that the flow conservation law is satisfied (i.e. all traffic injected into the network is delivered at the corresponding destination). In addition, we define the flow vector $\mathbf{x}^{(d)} \in \mathbb{R}_{+}^{L}$ as the vector with elements $x^{(d)}(\ell)$ containing the amount of flow in link $\ell$ destined to node $d$. Note that the flow conservation low tells us that the flow coming into any intermediate node needs to equal the flow going out of that node (unless it is its final destination),

$$
\mathbf{A} \mathbf{x}^{(d)}=\mathbf{s}^{(d)}, \text { for } d=1, \ldots, D .
$$

According to (2), each link $\ell$ needs to support the following amount of traffic $t_{\ell}=\sum_{d=1}^{D} x^{(d)}(\ell)$. General flow assignment techniques assume that the capacity of the link is high enough to carry such amount of traffic. However, the link capacity $c_{\ell}$ strongly depends on the radio resources allocated to that link (e.g. the operating frequency, the bandwidth, the transmit power, etc) and the interference environment. In this paper, we focus on the transmit power and thus the capacity of the link can be expressed as,

$$
c_{\ell}(\mathbf{P})=\ln \left(1+\operatorname{SINR}_{\ell}(\mathbf{P})\right),
$$

where $\operatorname{SINR}_{\ell}(\mathbf{P})$ denotes the Signal-to-Interference plus Noise Ratio of the $\ell$-th link and $\mathbf{P}=\left[\begin{array}{lll}p_{1} & \cdots & p_{L}\end{array}\right]$ represents the transmit power vector. Therefore, the capacity constraints $t_{\ell} \leq$ $c_{\ell}(\mathbf{P}), \ell=1, \ldots, L$, should be satisfied.

In the considered integrated backhaul network, we have two types of links, i.e. terrestrial and satellite links. Assuming the spectrum sharing between both, terrestrial receivers collect interference from satellite Earth-to-Space links in the $27.5-29.5 \mathrm{GHz}$ band. In addition, aggressive frequency re-use among terrestrial backhaul links is assumed to boost the spectral efficiency of the system. As a consequence, the interference power seen by the link $\ell \in \mathcal{T}$, being $\mathcal{T}$ the set of terrestrial links, is the sum of the power received from the terrestrial emitters and neighboring uplink satellite links operating in the same carrier frequency. Therefore, the corresponding $\operatorname{SINR}_{\ell}(\mathbf{P})$ is given by,

$$
\begin{aligned}
& \operatorname{SINR}_{\ell}(\mathbf{P})= \\
& \frac{p_{\ell} \cdot h_{\ell, \ell, k}}{\sum_{\substack{i \in \mathcal{T}(k) \\
i \neq \ell}} p_{i} \cdot h_{\ell, i, k}+\sum_{j \in \mathcal{S}_{U P}(k)} p_{j} \cdot h_{\ell, j, k}+\sigma_{\ell}^{2}}, \\
& \ell \in \mathcal{T}
\end{aligned}
$$

where $\mathcal{T}(k)$ and $\mathcal{S}_{U P}(k)$ denote the set of terrestrial links and satellite uplinks operating at frequency $k, h_{\ell, x, y}$ denotes the channel coefficient between the $x$-th link' transmit node and the $y$-th link' receive node, respectively, for a certain frequency $k$, and $\sigma_{\ell}^{2}$ is the noise power at the $\ell$-th link' receiver, which in this case is a terrestrial receiver.

Regarding the satellite links, the uplink is assumed to be interference-free, since the satellite is assumed to be in GEO orbit at $35,786 \mathrm{~km}$ from Earth. The huge propagation distance together with the regulations in the maximum EIRP density of current Ka band satellite systems is the reason why the satellite downlink is assumed to not interfere with the terrestrial links. However, the satellite downlink in the $17.7-19.7 \mathrm{GHz}$ band may receive harmful interference from terrestrial links and shall be duly considered. To do so, the SINR of the satellite downlinks, i.e. $\ell \in \mathcal{S}_{D L}$, can be expressed as follows,

$$
\operatorname{SINR}_{\ell}\left(\mathbf{P}_{\ell}\right)=\frac{p_{\ell} \cdot h_{\ell, \ell, k}}{\sum_{i \in \mathcal{T}(\ell)} p_{i} \cdot h_{\ell, i, k}+I_{\mathrm{co}, \ell}+\sigma_{\ell}^{2}}, \ell \in \mathcal{S}_{D L}
$$

where $\mathcal{T}(k), h_{\ell, \ell, k}$ and $h_{\ell, i, k}$ follow the same definition as in (4). Note that $\sigma_{\ell}^{2}$ is the noise power, in this case seen at the satellite dish antenna. The term $I_{\mathrm{co}, \ell}$ accounts for the interference received from adjacent satellite beams, which is not negligible in multi-beam satellite systems.

\section{Iterative Joint Power And Flow Assignment}

In this paper, the main objective is to obtain the optimal joint power control and flow assignment that maximizes the sum throughput of the network, i.e. $\sum_{d=1}^{D} \sum_{\substack{n=1 \\ n \neq d}}^{N}\left(s^{(d)}(n)\right)$. In addition, a penalty factor is included in the problem formulation to account for the cost of using the satellite links. 


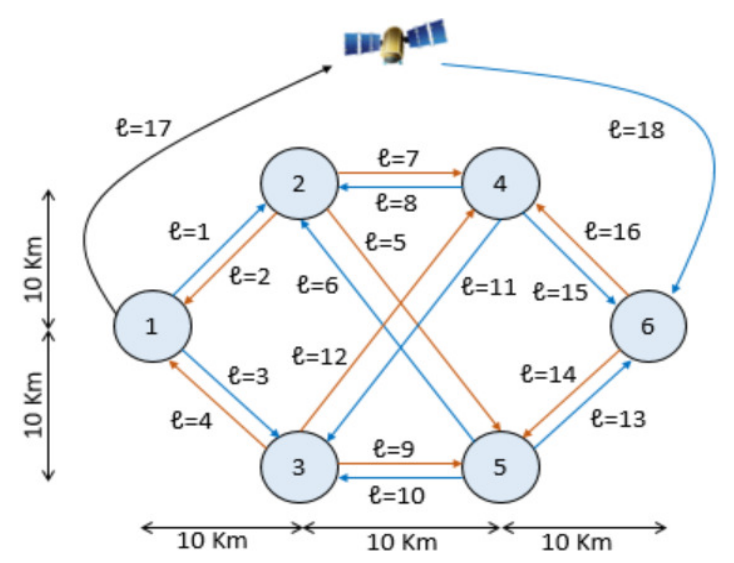

Fig. 1: Topology under evaluation including the frequency allocation represented by blue, brown and black arrows

The considered optimization problem is detailed below as,

$$
\begin{array}{ll}
\max _{\{\mathbf{x}, \mathbf{s}, \mathbf{P}\}} & \sum_{\substack{d=1 \\
\text { s.t. }}}^{D} \sum_{\substack{n=1 \\
n \neq d}}^{N} s^{(d)}(n)-\lambda \sum_{d=1}^{D} \sum_{\ell \in \mathcal{S}} x^{(d)}(\ell) \\
& \mathbf{A x} \mathbf{x}^{(d)}=\mathbf{s}^{(d)}, \quad d=1, \ldots, D \\
& \mathbf{x}^{(d)} \succcurlyeq 0, \quad d=1, \ldots, D \\
& \sum_{d=1}^{D} x^{(d)}(\ell) \leq c_{\ell}(\mathbf{P}), \quad \ell=1, \ldots, L \\
& 0 \leq p_{\ell} \leq p_{\ell}^{\max }, \quad \ell=1, \ldots, L
\end{array}
$$

where $\lambda \in \mathbb{R}_{+}$is the penalty factor and $p_{\ell}^{\max }$ accounts for the maximum transmit power of link $\ell$. The capacity constraints in (6) are not jointly convex due to presence of interference. Therefore, the problem is a difficult non-linear optimization problem and finding the global optimal solution is very challenging.

Exploiting conventional power control theory [16], we make use of the high SINR approximation in order to convert the difficult problem in (6) into a class of non-linear optimization called Geometric Programming (GP). In particular, we relax the capacity constraints as follows,

$$
c_{\ell}(\mathbf{P})=\ln \left(1+\operatorname{SINR}_{\ell}(\mathbf{P})\right) \approx \ln \left(\operatorname{SINR}_{\ell}(\mathbf{P})\right) .
$$

The high SINR regime can be achieved by carefully designing the carrier-per-link allocation [8]. However, this is not enough to transform the problem into GP. In order to convert the sumthroughput of the objective function in (6) into a posynomial, we use the inverse of the SINR in the $c_{\ell}(\mathbf{P})$ expressions,

$$
\begin{array}{r}
c_{\ell}(\mathbf{P}) \approx \\
-\ln \left(\sum_{\substack{i \in \mathcal{T}(k) \\
i \neq \ell}} \frac{h_{\ell, i, k}}{h_{\ell, \ell, k}} p_{i} p_{\ell}^{-1}+\sum_{j \in \mathcal{S}_{U P}(k)} \frac{h_{\ell, j, k}}{h_{\ell, \ell, k}} p_{j} p_{\ell}^{-1}\right. \\
\left.+\frac{\sigma_{\ell}^{2}}{h_{\ell, \ell, k}} p_{\ell}^{-1}\right), \ell \in \mathcal{T}
\end{array}
$$

for the terrestrial SINR expression in (4), and

$$
\begin{aligned}
& c_{\ell}(\mathbf{P}) \approx \\
& \quad-\ln \left(\sum_{i \in \mathcal{T}(\ell)} \frac{h_{\ell, i, k}}{h_{\ell, i, k}} p_{i} p_{\ell}^{-1}+\frac{I_{\mathrm{co}, \ell}+\sigma_{\ell}^{2}}{h_{\ell, \ell, k}} p_{\ell}^{-1}\right), \ell \in \mathcal{S}_{D L}
\end{aligned}
$$

for the satellite SINR expression in (5).

Finally, the optimization problem (6) with the relaxed expressions of $c_{\ell}(\mathbf{P})$ given in (8) and (9) can be transformed into a convex program by the change of variable $P_{x}=\exp \left(Q_{x}\right)$. As a consequence, a globally optimal solution can be efficiently obtained for the proposed approximate problem, for instance with the popular Interior Point or Barrier methods [17]. However, the constraint $t_{\ell} \leq \ln \left(\operatorname{SINR}_{\ell}(\mathbf{P})\right)$ imply that links with SINR $=1$ have zero capacity, but are allocated non-zero powers. To overcome this issue, in this paper we propose an iterative approach where the convexified version of the problem (6) is iteratively solved, by redefining after each iteration the node-link incidence matrix A. In particular, $\mathbf{A}^{(t+1)}$ is defined to be exactly the same as $\mathbf{A}^{(t)}$ but removing the links that have been assigned very low traffic flow (because SINR is low). In other words, at each iteration the incidence matrix is redefined as follows,

$$
A^{(t+1)}(\ell, n)= \begin{cases}A^{(t)}(\ell, n) & \text { if } \operatorname{SINR}_{\ell}>\beta \\ 0 & \text { otherwise, }\end{cases}
$$

where $\beta$ is a parameter that needs to be adjusted depending on the considered network. In this paper, we fixed $\beta=1.5$.

In addition, since our objective function is maximization of throughput, it may occur that some links with good SINR are assigned very limited flow at the expenses of certain transmit power. One way to avoid spending power to transmit limited throughput is to run an additional iteration where the following links are removed,

$$
A^{(t+1)}(\ell, n)= \begin{cases}A^{(t)}(\ell, n) & \text { if } \sum_{d=1}^{D} x^{(d)}(\ell)>\eta \\ 0 & \text { otherwise. }\end{cases}
$$

Similarly, $\eta$ needs to be adjusted depending on the considered network. In this paper, we fixed $\beta=0.01$. Note that the using the topology redefinition in (10), the objective function always improves with the iterations. Also, the procedure in (10) guarantees the existence of a feasible solution. However, we cannot provide the same guarantees with (11).

\section{Simulation Results}

For illustration purposes, we will demonstrate the benefits of the proposed iterative approach with a small scale wireless backhaul topology as the one illustrated in Fig. 1, with $N=7$ nodes and $L=18$ directed links. Note that node number 7 represents the satellite and we assume that node 1 and node 6 have hybrid terrestrial-satellite transmission capabilities. For the sake of simplicity, we assume a single traffic flow, which goes from node 1 to node 6 . The goal is to maximize the throughput going from node 1 to node 6 .

We assume a fix frequency allocation, where all links except the satellite uplink operate under an aggressive frequency reuse 

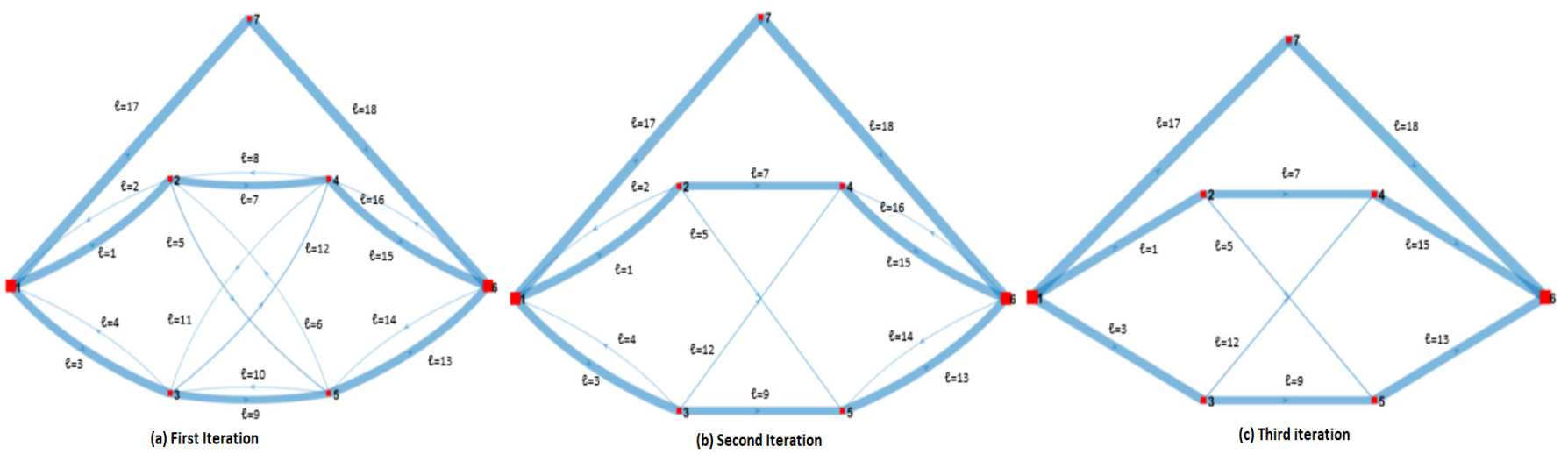

Fig. 2: Illustration of link removal procedure and the resulting flow assignment $(\lambda=0.49)$

TABLE I: Simulation Parameters

\begin{tabular}{|c|c|}
\hline \multicolumn{2}{|c|}{ Terrestrial Segment } \\
\hline Parameter & Value \\
\hline Antenna pattern & ITU-R F.1245-2 \\
Max. antenna gain & $24.5 \mathrm{dBi}$ \\
Max. transmit power & $-20.6481 \mathrm{dBW}$ \\
Channel & LoS channel (path loss) \\
Noise power & $-121.52 \mathrm{dBW}$ \\
Antenna height & Between 13 and $48 \mathrm{~m}$ \\
\hline \multicolumn{2}{|c|}{ Satellite Segment } \\
\hline Parameter & Value \\
\hline Satellite location & GEO 13 ${ }^{\circ} \mathrm{E}$ \\
Satellite gain & $55.77 \mathrm{dBi}$ \\
Dish antenna pattern & ITU-R S.465 \\
Max. dish antenna gain & $42.1 \mathrm{dBi}(90 \mathrm{~cm}$ dish) \\
Co-channel interference & $-113 \mathrm{dBW}$ \\
Max transmit power from Earth & $7.9 \mathrm{dBW}$ \\
Max transmit power from Satellite & $9.23 \mathrm{dBW}$ \\
Channel & LoS channel (path loss) \\
Noise power & $-126.94 \mathrm{dBW}$ \\
Dish antenna height & Between 13 and $48 \mathrm{~m}$ \\
\hline
\end{tabular}

scheme assuming the availability of 2 carriers of $56 \mathrm{MHz}$ each. The uplink is assumed to be in an interference-free carrier for simplicity. Fig. 3 shows a channel matrix instance for the considered topology, where the diagonal elements correspond to the intended links, while the off-diagonal elements correspond to non-desired interference links. It can be observed that due to the aggressive frequency reuse, many non-diagonal elements are significantly high. Note that link 17 and link 18 represent the satellite uplink and downlink, respectively. The values depicted in Fig. 3 have been obtained with the simulation parameters given in Table I.

To validate the proposed approach, we first run an experiment considering $\lambda=0.49$ (we will discuss later in the paper on how to obtain this variable) and the solution is achieved in only 3 iterations. The result of the first iteration, with $L=18$, is illustrated in Fig. 2(a), where the obtained flow assignment per link is illustrated by making the width of the links proportional to the carried flow. In this case, the total throughput that is transmitted from node 1 to node 6 is $10.0424 \mathrm{~b} / \mathrm{s} / \mathrm{Hz}$ and the total transmitted power in the terrestrial segment is $100.11 \mathrm{~mW}$. Next, we remove links 6 ,

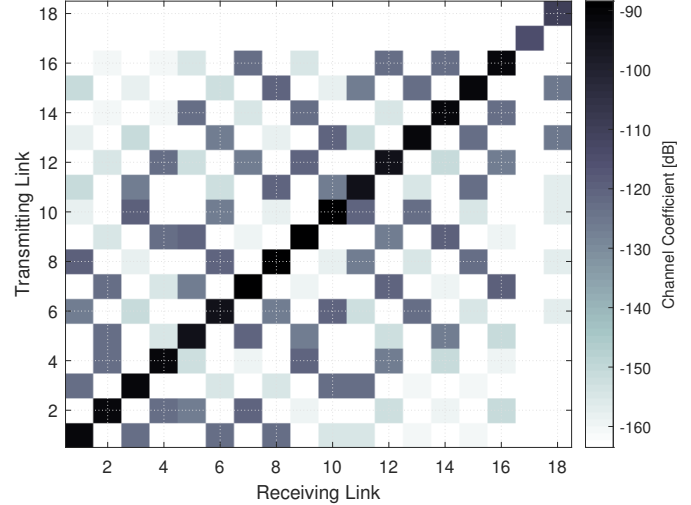

Fig. 3: Channel coefficients for the assumed frequency assignment

8, 10 and 11, which have SINR close to 1 and we repeat the optimization with the new topology. The flow assignment after the second iteration is depicted in Fig. 2(b), where the total delivered throughput has been slightly improved (i.e. $10.0483 \mathrm{~b} / \mathrm{s} / \mathrm{Hz}$ ) and a reduction on the transmitted power due to the link removal procedure has been achieved (total transmitted power in the terrestrial segment is now $94.16 \mathrm{~mW}$ ). Finally, an addition iteration is performed according to (11), and the resulting topology and flow assignment is shown in Fig. 2(c). The total delivered throughput remains the same as in the previous iteration but the total transmitted power in the terrestrial segment is reduced to $65.46 \mathrm{~mW}$ by further removing links 2, 4, 14 and 16. Fig. 4 shows the evolution of SINR and power assignment over the 3 iterations. Note that for the sake of clarity, we have only included the terrestrial links in Fig. 4, as the power for satellite links is much higher.

Next, we study the penalty parameter $\lambda$ for the small scale network illustrated in Fig. 1. Incorporating a penalization term into our objective function provides a design parameter for the operators to decide how much flow to be sent though the satellite depending on the cost of using such links. Fig. 5 shows the amount of traffic flow that is routed through the satellite segment and the overall throughput delivered at the destination node 6 , respectively, as a function of the penalty factor $\lambda$. It can be observed that when the cost of using the 

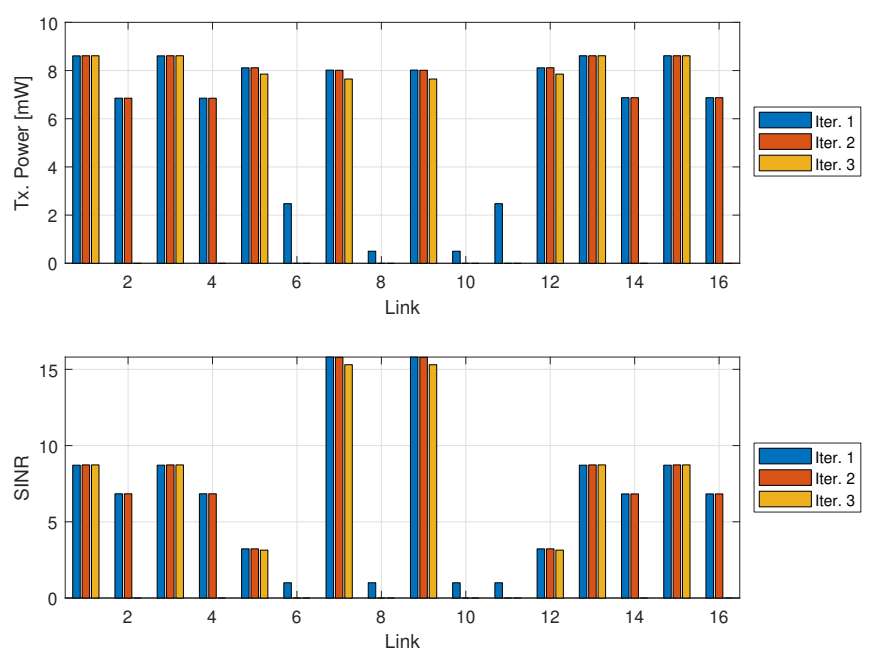

Fig. 4: SINR and power evolution for the terrestrial links over the iterative procedure

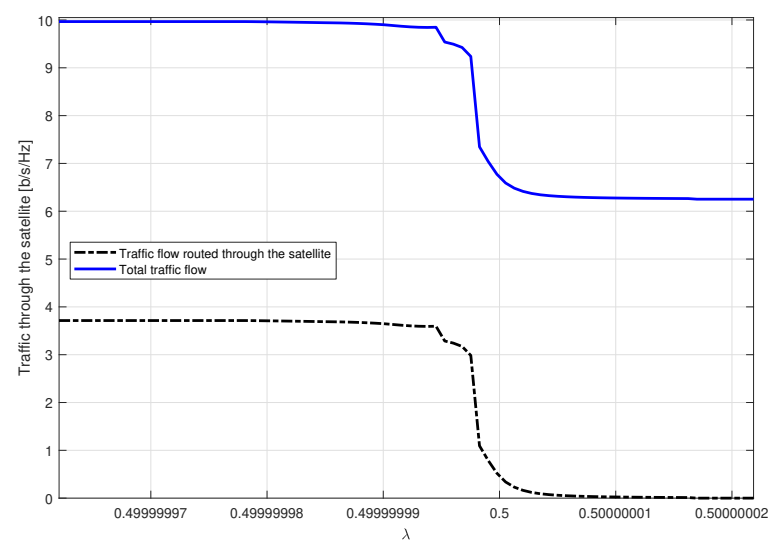

Fig. 5: Delivered traffic flow as a function of $\lambda$

satellite links is high, all the traffic is routed via the terrestrial network, achieving a total throughput of $6.25 \mathrm{~b} / \mathrm{s} / \mathrm{Hz}$. When the cost of using the satellite is reduced, e.g. $\lambda$ decreases, and the satellite links are used to offload the terrestrial segment. In this case, the total throughput that can be delivered increases to $10.04 \mathrm{~b} / \mathrm{s} / \mathrm{Hz}$, which represents a $60 \%$ increase with respect to the terrestrial-only counterpart.

\section{CONClusion}

In this paper, we presented an iterative joint power control and flow assignment algorithm for integrated satelliteterrestrial networks with aggressive frequency reuse and spectral coexistence between terrestrial and satellite segments. By using a high SINR approximation and transforming the capacity expressions into posynomials in $\mathbf{P}$, we obtained a GP problem which can be efficiently solved to global optimality. In order to deal with the limitations introduced by the high SINR approximation, we proposed a link-removal procedure which removes those links with SINR close to 1 . To further improve the power efficiency, we complemented the linkremoval procedure with an additional iteration that suppresses any link with residual flows. Results based on numerical simulations confirmed the potential of integrated satelliteterrestrial backhaul network under the spectral coexistence assumption by considering the proposed iterative approach. In future works, the joint frequency, power and flow assignment would be investigated.

\section{ACKNOWLEDGMENT}

This work was partially supported by the Luxembourg National Research Fund (FNR) under the CORE project "ROSETTA" (11632107), the CORE project "ProCAST", and the ANR-FNR project "SIERRA" - Spectral efficient receivers and resource allocation for cognitive satellite communications.

\section{REFERENCES}

[1] Ericsson, $2018, \quad \mathrm{https} / / \mathrm{www} \cdot$ ericsson.com/assets/local/microw
outlook/documents/ericsson-microwave-outlook-report-2018.pdf.

"Ericsson Microwave Outlook," Dec.

[2] A.H. Sherin, H.S. Hassanein, and G. Takahara, Routing for Wireless Multi-Hop Networks, Springer-Verlag, New York, USA, 2013.

[3] T. Meng, F. Wu, Z. Yang, G. Chen, and A.V. Vasilakos, "Spatial Reusability-Aware Routing in Multi-Hop Wireless Networks," IEEE Trans. Comput., vol. 65, no. 1, pp. 244-255, Jan. 2016.

[4] J. Nuñez Martínez and J. Mangues, "A Survey on Routing Protocols that really exploit Wireless Mesh Networks," Journal of Communications, Academy Publisher, vol. 5, no. 3, pp. 211-231, Mar. 2010.

[5] G. Parassidis, "Interference-Aware Routing in Wireless Multihop Networks," PhD, ETH Zurich, Switzerland, 2008.

[6] Q. Zhu, Z. Yuan, J. Song, Z. Han, and T. Basar, "Interference Aware Routing Game for Cognitive Radio Multi-Hop Networks," IEEE J. Sel. Areas Commun., vol. 30, no. 10, pp. 2006-2015, Nov. 2012.

[7] F. Mendoza, R. Ferrús, and O. Sallent, "Flexible Capacity and Traffic Management for Hybrid Satellite-Terrestrial Mobile Backhauling Networks," International Symposium on Wireless Communication Systems (ISWCS), Poznan, Poland, Sep. 2016.

[8] E. Lagunas, S. Maleki, L. Lei, C. Tsinos, S. Chatzinotas, and B. Ottersten, "Carrier Allocation for Hybrid Satellite-Terrestrial Backhaul Networks," ICC Workshop on Satellite Communications: Challenges and Integration in the $5 G$ ecosystem, Paris, France, May 2017.

[9] M. Corici et al., "SATis5: A 5G Testbed Integrating Satellite and Terrestrial Infrastructures," Advanced Satellite Multimedia Systems Conference (ASMS), Berlin, Germany, Sept. 2018.

[10] S. Chatzinotas et al., "Cognitive Approaches to Enhance Spectrum Availability for Satellite Systems," Int. J. Satellite Commun. and Networking, Nov. 2016.

[11] M. Shaat, A. Pérez-Neira, G. Femenias, and F. Riera-Palou, "Joint frequency assignment and flow control for hybrid terrestrial-satellite backhauling networks," International Symposium on Wireless Communication Systems (ISWCS), Bologna, Italy, Aug. 2017.

[12] P. Moberg, "Simultaneous Routing and Resource Allocation in Multihop Wireless Networks using Optimization," IEEE Global Telecommun. Conf. (GLOBECOM), San Francisco, CA, USA, Nov. 2006.

[13] L. Xiao, M. Johansson, and S.P. Boyd, "Simultaneous Routing and Resource Allocation via Dual Decomposition," IEEE Trans. Commun. vol. 52, no. 7, pp. 1136-1144, Jul. 2014.

[14] R. Di Taranto and H. Wymeersch, "Simultaneous Routing and Power Allocation using Location Information," ASILOMAR Conf. in Signals, Systems and Computers, Pacific Grove, CA, USA, Nov. 2013.

[15] L. Rosati and G. Reali, "Jointly Optimal Routing and Resource Allocation in Hybrid Satellite/Terrestrial Networks," Int. Workshop in Satellite and Space Communications, Madrid, Spain, Sept. 2006.

[16] M. Chiang, C.W. Tan, D.P. Palomar, D. O'neil, and D. Julian, "Power Control By Geometric Programming," IEEE Trans. Wireless Commun., vol. 6, no. 7, pp. 2640-2651, Jul. 2007.

[17] S. Boyd and L. Vandenberghe, Convex Optimization, Cambridge University Press, 2004 